\title{
Resonant frequency stabilization technique in series-series contactless energy transfer systems
}

\author{
M. Marcinek, M. Holub, S. Kalisiak, R. Palka \\ West Pomeranian University of Technology \\ Department of Power Engineering and Electrical Drives \\ Sikorskiego 37, 70-313 Szczecin, Poland \\ e-mail:marcinek@zut.edu.pl
}

(Received: 28.11.2016, revised: 17.03.2017)

\begin{abstract}
A technique for stabilization of resonant frequency is proposed in this paper. An additional power circuit, a compensator that allows keeping constant resonance frequency was introduced by the authors. In the presented solution the resonant circuit frequency stabilization is achieved by forcing a zero phase shift between the current and the voltage of the main switching module.
\end{abstract}

Key words: contactless energy transfer, frequency stabilization, inductive energy transfer, mutual coupled coils, resonant inverters, wireless energy transmission

\section{Introduction}

Contactless energy transfer systems are becoming more and more popular as a way for recharging of batteries of mobile electronics (cell phones, tablets etc.) [1,2]. Different solutions which are in everyday use as home accessories are available on the market. In the same time hybrid and electric vehicles are intensively researched. EV technology becomes a serious alternative for internal combustion vehicles, because of zero emission or less noise. This solution can also be applied in other transport systems using different linear drives [3, 4].

However, this technology has important drawbacks like limited capacity of the batteries, necessity of wired connection between the vehicle and the supply grid or mandatory idle time during recharge. In area of ICET (Inductive Contactless Energy Transfer) technology several solutions were developed to overcome those drawbacks, for instance solution to deliver power during short stops or in-motion mode [5-8]. It is important to maximize the energy transmission, for that reason optimization of the construction of coils and the capability to transfer energy, when those are in misalignment, are investigated [8-11].

On the other hand, to keep demanded levels of power in the load, additional circuits or the control algorithms were introduced. The simplest idea is to follow the resonance point using, 
for instance, Phase Locked Loop [12]. Another way is to dynamically tune or detune from/to resonance with usage of auxiliary circuits. From literature review it can be seen that both capacitors and inductors are used [13-17]. In this paper a solution of the inductive auxiliary circuit is presented. In this paper we present a deeper analysis of the influence of mutual coupling and load change to voltage - current phase shift $\phi$. In addition a set of equations for arbitrary configuration of the system's parameters is introduced. Investigations were performed for two cases: with an active compensation circuit and without it. To conduct simulations and experiments previously presented topology and control the strategy $[18,19]$ of the compensator were used.

\section{Proposed ICET system construction}

In this paper a series-series topology of ICET was used. It consists of an H-bridge switching circuit and resonant circuits (built of a series connection of a winding of a transformer with a resonant capacitor). On the secondary side a Graetz bridge and an adjustable resistor were used. In a red rectangle the auxiliary compensating circuit is marked. It is connected in parallel to the resonant capacitor on the primary side of the system. It consists of two branches. Each of them is made of a series connection of a power transistor, an inductor and a diode. The proposed topology is shown in Fig. 1(a).

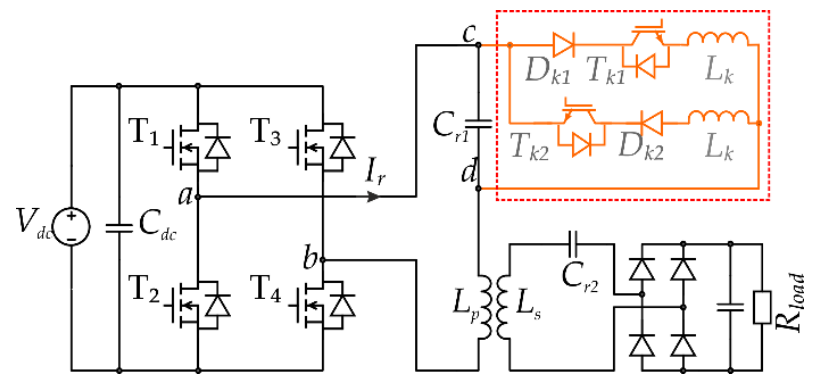

(a)

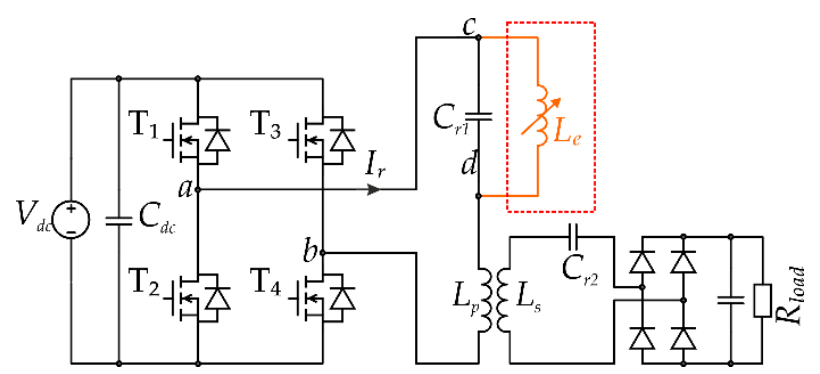

(b)

Fig. 1. The proposed topology of ICET system with the compensating circuit: full schematic (a); simplified compensator circuit (b) 
The principle of the operation is like similar constructions found in the literature $[12,20$, 21]. The H-bridge produces a square voltage wave on its output, which feeds the resonant circuit on the primary side $-V_{a b}$. Then, trough magnetic coupling between $L_{p}$ and $L_{s}$, energy is transferred to the secondary side. On the primary side an additional compensator must be synchronized with the voltage $V_{r}$ on the $C_{r 1}$ capacitor. Detailed description and proposed control procedure of such a compensator was previously presented in $[18,19]$.

Just few additional remarks must be stated regarding the aforesaid contactless energy transfer system. First of all no ferrite core was used in the set of the coils. Primary (P) and secondary (S) sides are not mechanically connected. In consequence such arrangement has a large number of degrees of freedom. It is one of the most important features, but also a major issue from control system point of view. The relative position between coils may be freely changed - a mutual coupling factor $k$ is related to it. The coupling can be changed due to a horizontal, vertical or angular misalignment. Such possibilities are depicted in Fig. 2.

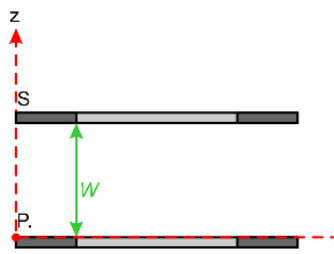

(a)

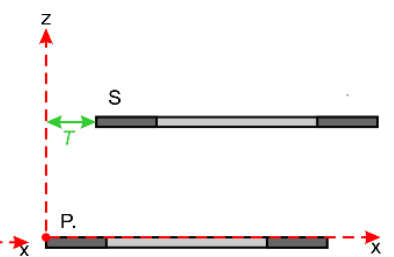

(b)

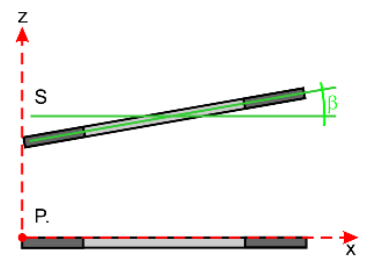

(c)

Fig. 2. Different coupling relations corresponding to different relative positions between a transmitting (P) and a receiving (S) coils: (a) the vertical misalignment; (b) the horizontal misalignment; (c) the angular misalignment

Secondly, load applied to the system can vary in time. For instance, in the case of battery chargers, the biggest demand on power is when the battery is almost depleted (for Li-Ion battery - constant current mode), Fig. 3. In contrast, when a battery charger is in constant voltage mode less power is demanded. In [6] a system for a continuous roadway supply of EV is presented. Authors show that amount of power that must be transferred to the vehicle is related to its speed. For simulations and for the needs of experiments these phenomena can be simplified and represented by a lumped, variable load resistance.

\section{Simulations and mathematical description}

The system in Fig. 1(a) can be simplified to an equivalent form found in Fig. 1(b), where the compensator may be replaced by a single, tunable inductance $L_{e}$. 


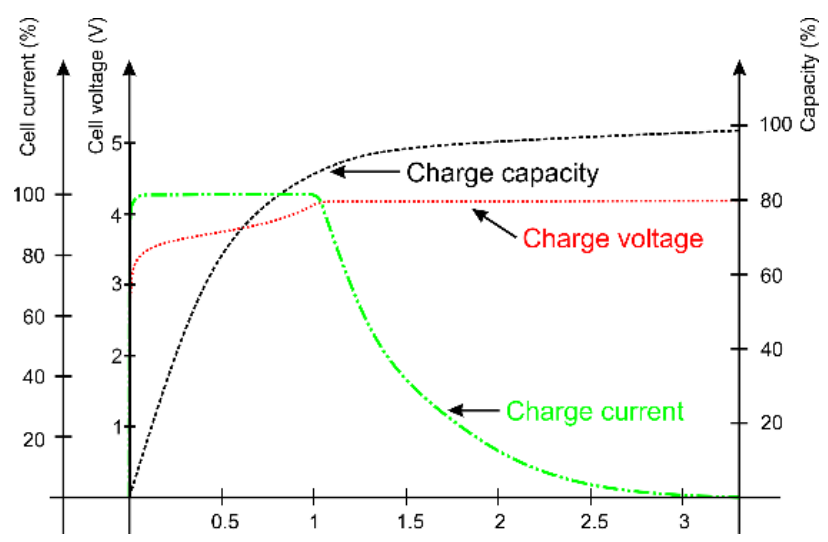

Fig. 3. Single cell time dependency of the charge process

In order to conduct simulations in AC sweep domain, the circuit depicted in Fig. 1(b) was further simplified to the form shown in Fig. 4. Magnetically coupled coils $\left(L_{p}\right.$ and $\left.L_{s}\right)$ are replaced by an equivalent circuit of a transformer - T. Output circuit consists of the Graetz rectifier bridge, the filtering capacitor and load resistance. For the purpose of analysis it was replaced by an equivalent resistance $R_{l}$. it is expressed as $R_{l}=0.811 \cdot R_{\text {load }}$. This simplification is valid only when a current conducted through a rectifier is continuous and it has a sinusoidal shape. If above conditions are fulfilled the system could be analyzed with first harmonic approximation $[22,23]$ and it may be represented by an equivalent circuit depicted in Fig. 4.

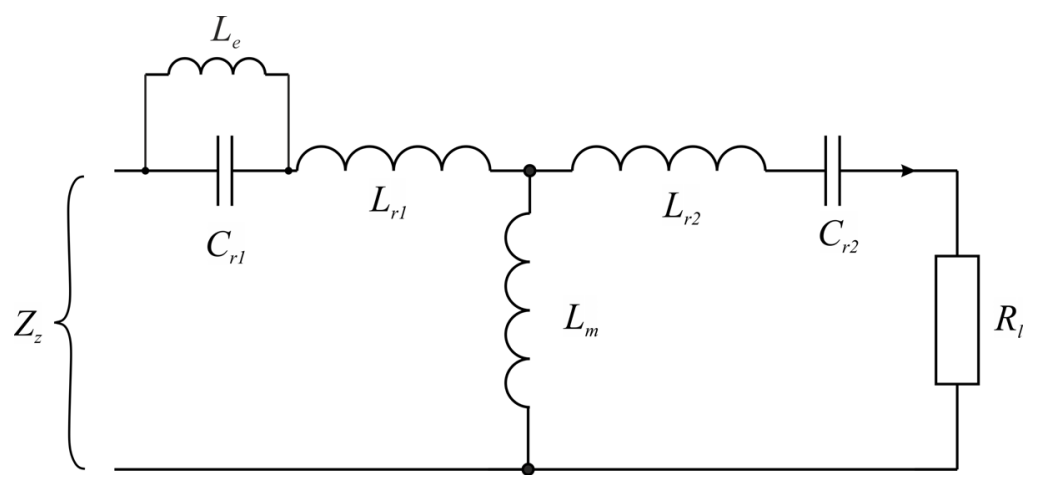

Fig. 4. Simplified equivalent circuit of the proposed ICET system

We start the analysis by assuming that the additional circuit is switched off and the system is in a non-nominal operating point. It means that inductance $L_{e}$ is disconnected from capacitor $C_{r 1}$, or it has infinite value of inductance. Instantaneous value of impedance $Z_{z}$ seen on the output of a switching circuit can be expressed as (1). In result, an instantaneous phase shift can be calculated (2). The considered phase shift is estimated between an output voltage of a H-bridge and its current. The non-nominal operating point will cause non-resonant switching of transistors and it will lead to the increase of amount of generated losses. In such condition 
the simplest solution is to change the switching frequency of inverter's switches [12]. However, in many applications it is unacceptable, because of a wide frequency range of radiated electromagnetic interferences.

$$
\begin{gathered}
Z_{z}=\frac{\omega^{2} L_{m}^{2} R_{l}}{R_{l}^{2}+\left(\omega L_{m}+\omega L_{r 2}-\frac{1}{\omega C_{r 2}}\right)^{2}}+j\left[\omega L_{r 1}+\omega L_{m}-\frac{1}{\omega C_{r 1}}-\frac{\omega^{2} L_{m}^{2}\left(\omega L_{m}+\omega L_{r 2}-\frac{1}{\omega C_{r 2}}\right)}{R_{l}^{2}+\left(\omega L_{m}+\omega L_{r 2}-\frac{1}{\omega C_{r 2}}\right)^{2}}\right], \\
\phi=\arctan \left[\frac{R_{l}^{2}\left(\omega^{2} L_{r 1} C_{r 1}+\omega^{2} L_{m} C_{r 1}-1\right)-\omega^{3} L_{m}^{2} C_{r 1} Z_{s}+\omega^{2} C_{r 1}\left(L_{r 1}+L_{m}\right) Z_{s}^{2}-Z_{s}^{2}}{\omega^{3} L_{m}^{2} C_{r 1} R_{l}}\right],
\end{gathered}
$$

where:

$$
Z_{s}=\omega L_{m}+\omega L_{r 2}-\frac{1}{\omega C_{r 2}} .
$$

An exemplary range of a phase change $\phi$ is depicted in Figs. 5(a) and (b).

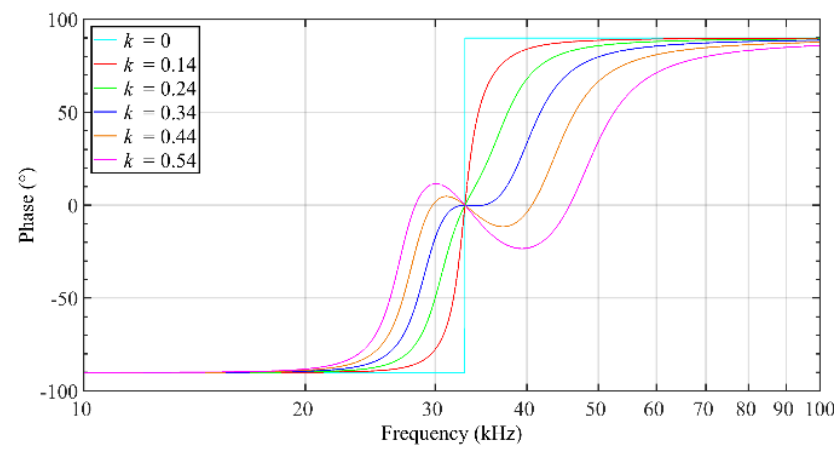

(a)

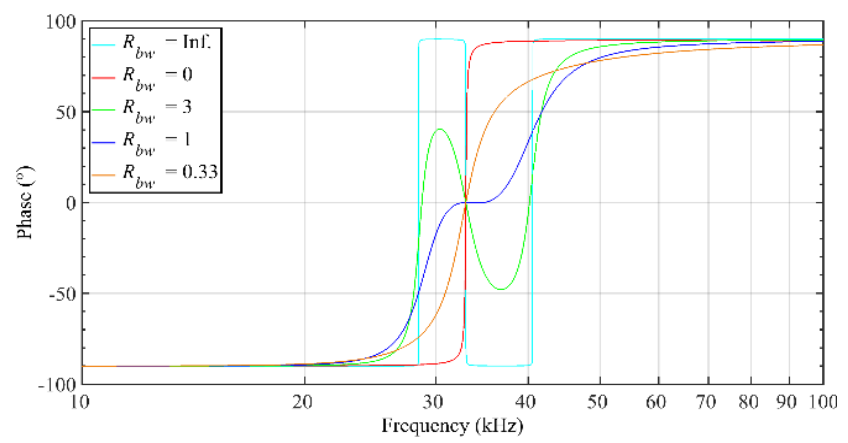

(b)

Fig. 5. Characteristics of the phase shift between the output H-bridge current $\left(I_{r}\right)$ and the output voltage $\left(U_{a b}\right)$ for the $k(a)$ and the load (b) change without active compensation 
Top (a) characteristics present response of system to the coupling coefficient change for the various frequencies (in range of 10 to $100 \mathrm{kHz}$ ), the bottom (b) one presents the response to the load change. $R_{b w}$ is the load factor, which is described as $R_{n o m} / R_{i}$. Resistance $R_{\text {nom }}$ was obtained when transistors were switched in a zero-current mode. $R_{i}$ represents the instantaneous value of load resistance. In the presented report the authors show that the transistors will be switched in the zero current mode, using a frequency $f_{s}$ of around $36.5 \mathrm{kHz}$.

In both cases it can be noticed that the phase has positive and negative values, moreover it is possible to achieve more than one point where $\phi$ has a value of zero.

From the results depicted in above figure it can be stated that in a series resonance ICET both the change of the value of the load or the mutual coupling factor will cause the change of the value of phase shift $\phi$ between the primary current and voltage. It is well known that in series resonant systems, a change of phase will cause operation in the so called capacitive or inductive mode. This leads to increased losses in power semiconductors as hard switching technique is used. The proposed compensator is able to work when frequency of the resonant circuit will drop below the switching frequency of the H-bridge. Such a scenario occurs when the value of the load or the mutual coupling factor will decrease.

\section{System with active frequency stabilization}

Depending on $k$ or $R_{l}$, the depth of the change of the value of $L_{e}$ must be adjusted. In the proposed compensator, conduction time of the transistors can be freely changed via the delay activation angle $-\alpha$ [14]. This change will influence the value of $L_{e}$ in the way that is presented in Fig. 6. To estimate the equivalent value of the inductance Equation (3) can be used $[17,24]$ :

$$
L_{e}=\frac{\pi L}{2 \pi-2 \alpha+\sin (2 \alpha)} .
$$

In $[17,24,25]$ a similar system was investigated. The main difference is that a centralized, single reactor was used. It was noticed that dividing it in to two branches will allow to increase the parameters of the range of the change. It is possible because in the proposed solution, an ignition angle in the range of $0 \leq \alpha \leq \pi / 2$ can be set. The resultant inductance value is equal to half of the self-inductances of the coils. It can be observed in Fig. 6(a) at an angle $\alpha$ of $0^{\circ}$.

$$
\phi=\arctan \left[\begin{array}{l}
\frac{\omega R_{l}^{2}\left(L_{r 1}+L_{e}+L_{m}+\omega^{2} L_{e} L_{m} C_{1}-\omega^{2} L_{e} C_{1} L_{r 1}\right)}{\omega^{2} L_{m}^{2} R_{l}-\omega^{4} L_{m}^{2} L_{e} C_{r 1} R_{l}}+\cdots \\
\cdots+\frac{\left(\omega L_{r 1}+\omega L_{m}-\omega^{3} L_{e} C_{r 1} L_{r 1}-\omega^{3} L_{m} L_{e} C_{r 1}\right) Z_{s}^{2}+\left(\omega^{4} L_{e} L_{m}^{2} C_{r 1}-\omega^{2} L_{m}^{2}\right) Z_{s}}{\omega^{2} L_{m}^{2} R_{l}-\omega^{4} L_{m}^{2} L_{e} C_{r 1} R_{l}}
\end{array}\right] .
$$




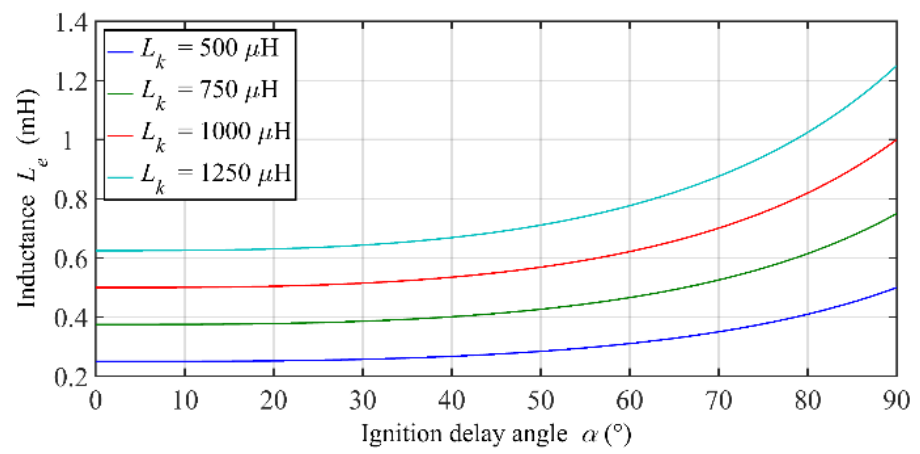

(a)

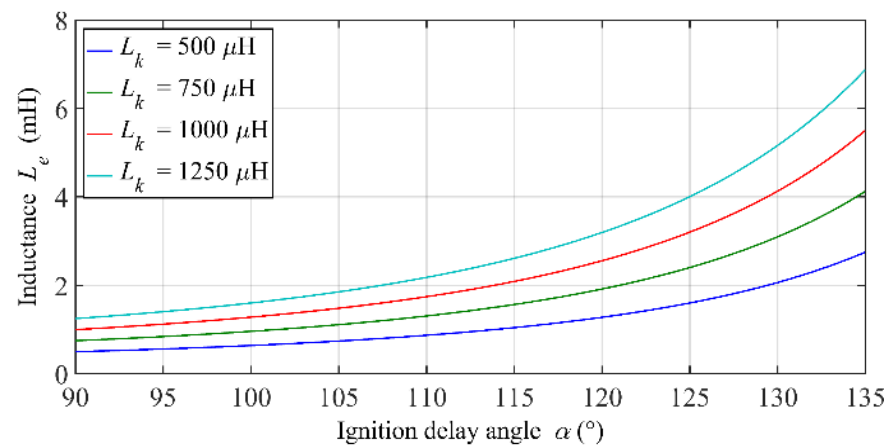

(b)

Fig. 6. Characteristics of an equivalent induction $L_{e}$ change related to ignition delay angle $\alpha$

The influence of additional inductance $L_{e}$ and results of AC simulations are presented in Fig. 7. A green line presents response of the system to nominal conditions, when zero current switching (ZCS) of the transistors is achieved. Then the operating point of the system was changed. Two points of work were simulated one for $k=0.34$ and $R_{b w}=0.33$ (a) and the other for $k=0.24$ and $R_{b w}=1$ (b). Each non-nominal state is presented as a red line. In both cases, the point of zero phase value was shifted into direction of smaller frequencies. By adjusting $L_{e}$ it is possible to keep zero value of phase shift at desired resonant frequency value (blue line). Instantaneous value of $\phi$ can be expressed by (4).

A remark to results shown in Fig. 5 and Fig. 7 should be given. They are valid in the entire operation range but only for an ideal scheme from Fig. 4 where the current is always continuous. In the case of Fig. 1(a), so wide range of the operation frequencies (like in AC simulations) shouldn't be used. According to [26] to use the simplified scheme the value of ratio of the resonant frequency to the switching frequency must be in the correct range. When this ratio has incorrect value - bellow unity - a current won't be continuous, thus the equivalent model of the load shouldn't be used. In the described state it is possible that in one half of the period of the output voltage $\left(V_{a b}\right)$, multiple half-waves (oscillations) of the current appear. In our case this region is located below frequencies of around $18-20 \mathrm{kHz}$. 


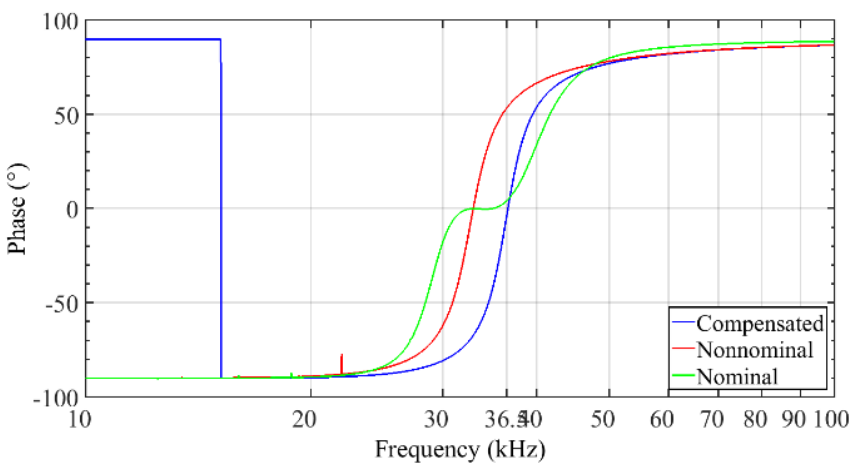

(a)

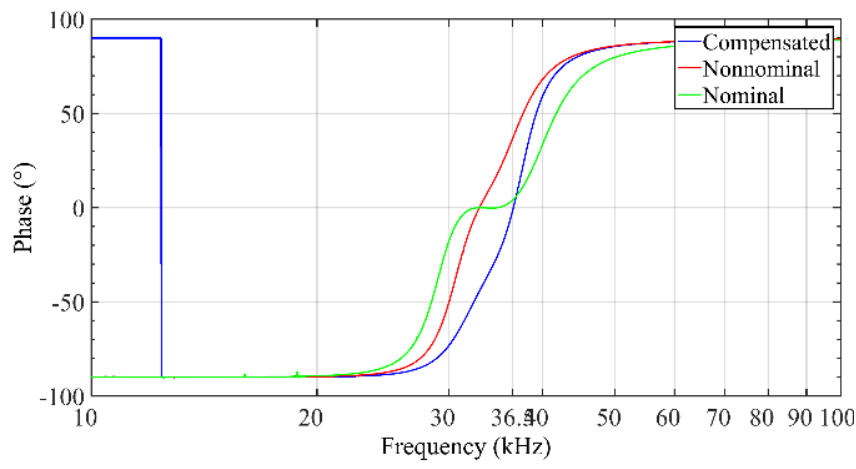

(b)

Fig. 7. Characteristics of phase shift between $I_{r}$ and $U_{A B}$ for the coupling $k$ (a) and the load (b) change with active compensation

\section{Laboratory stand}

The presented solution could be scaled and applied to the automotive industry, as an alternative way for the battery charger. As it was noticed in the introduction, a main drawback of such applications is unprecise alignment between a transmitter (A) and a receiver (B). For research purposes, a dedicated transformer was constructed (Fig. 8(a)). It allows for the movement up and down along the axis (D) of the receiver coil. Four guides are used to provide a centric movement of the receiver (C), to ensure repeatability of horizontal placement, wooden blocks are used.

An H-bridge and a capacitor were connected in series to the primary side of the transformer. Then, the secondary was built using a full bridge rectifier with a capacitive filter and a variable load resistor. In Fig. 8(b) the laboratory stand is presented. To acquire data from the laboratory stand, a digital oscilloscope Tektronix DPO 4054 with probes TCP0030A, P5205, also a power analyzer NORMA 5000 were used. 


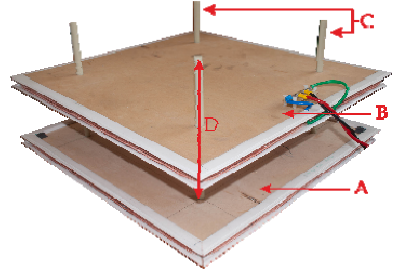

(a)

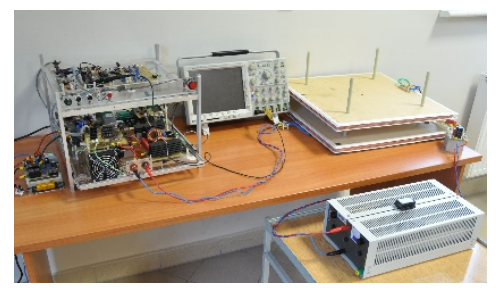

(b)

Fig. 8. Physical construction of a coreless transformer (a). View on the laboratory stand (b)

\section{Basic measurements results}

So far, influence of the proposed compensator on the resonant circuit parameters was presented. It was presented in Fig. 7 that for a change of the load or the coupling factor the supply inverter can operate with constant switching frequency and zero phase shift. Compensation of the changes of the operational point results in the change of the amount of power that was transmitted to the load. During the experiments coupling factor $k$ and load factor $R_{b w}$ were changed. The shape of the power map also changed. The map, when power inverter was working with the fixed frequency of transistors switching and no stabilization was applied is presented in Fig. 9(a). The achieved shape is nonlinear also it can be clearly seen that reducing $R_{b w}$ and $k$ will decrease the amount of power in the load. In part (b) the power transmitted to load was depicted. In those, the states change in the resonant frequency $f_{r}$ were compensated via the proposed circuit. The falling part of the map depicted in Fig. 9(b) is caused by the current limit in power source.

(a)

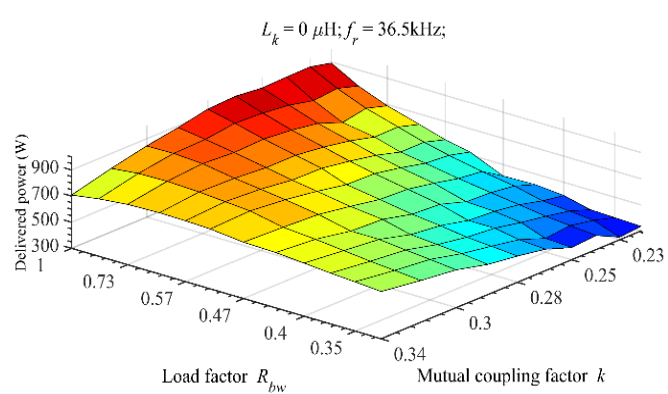

(b)

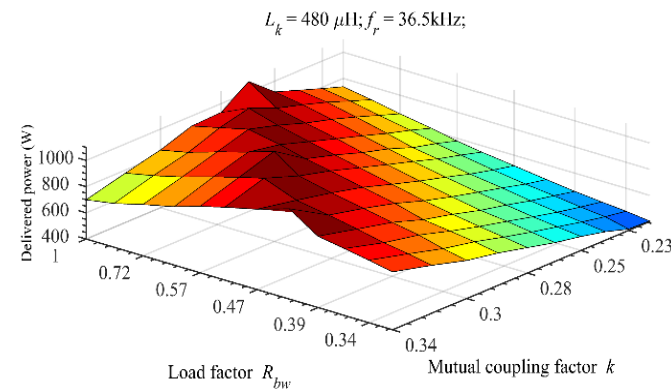

Fig. 9. Power delivered to load resistor with (a) and without (b) resonant frequency stabilization

Based on the presented results it can be stated that the transmission of fixed power value is possible when the operation point is changing. Noting from Fig. 9 two points have been selected. Let's analyze following parameter combinations: $R_{b w}=0.57$ with $k=0.32$ and $R_{b w}=0.41$ with $k=0.26$. In part (a) results without stabilization are depicted. Here $768 \mathrm{~W}$ and 
$570 \mathrm{~W}$ were transmitted to the load with above given parameter combinations, respectively. For the system operating with compensation and same parameter combinations $1 \mathrm{~kW}$ and $660 \mathrm{~W}$ were transmitted, (part (b)).

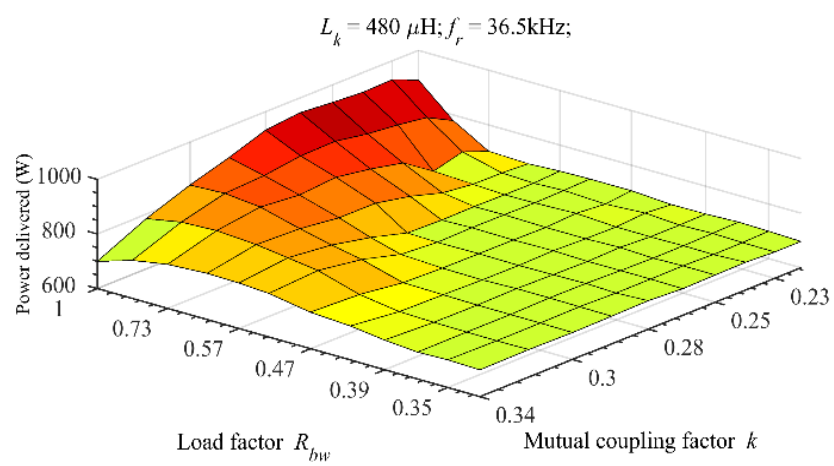

Fig. 10. A stabilization of the output power with usage of the proposed compensator

It should be pointed out, that for the second point the system has been working with an active input current limit still the power transmitted to the load was greater than in operating mode without additional stabilization. It proves, that the operation with the compensation, allows to transmit greater volume of power than in the nominal point. Moreover, it means that delivery of nominal power should be possible in a wider range of the system's parameters change. However, to stabilize it, phase adjustment is necessary. It conducts to operation with phase shift not equal to zero. Such a scenario is depicted in Fig. 10.

\section{Efficiency of the system}

Efficiency changes in the function of the output power and the coupling factor of the proposed system was investigated. During experiments the input voltage was adjusted to achieve $100 \mathrm{~V}$ at the output hence to set fixed output power as load resistance changed. The procedure was repeated for nine distances between coils of the transformer.

Fig. 11 depicts the achieved results, blue lines represent calculated $\eta$ without and red with an activated auxiliary circuit. Maximal efficiency exceeds $90 \%$ and was achieved in the nominal point, in contrast to the minimum load and minimal $k$ efficiency is around $62 \%$. It should be noticed that in both scenarios, the lines are on similar levels. It means that losses of the main H-bridge were reduced.

\section{Conclusions}

In this paper, a technique for stabilization of the resonant frequency was presented. The simulation results show that the change of the additional inductance $L_{e}$ allows to keep constant 
resonant frequency and to minimize phase shift. Such a type of operation (with frequency of the inverter equal to the resonant frequency of the circuit and zero phase shift) will have effect on the power transfer capability of the system. Based on this it is possible to propose output power stabilization technique when zero phase shift is not mandatory requirement to fulfil.

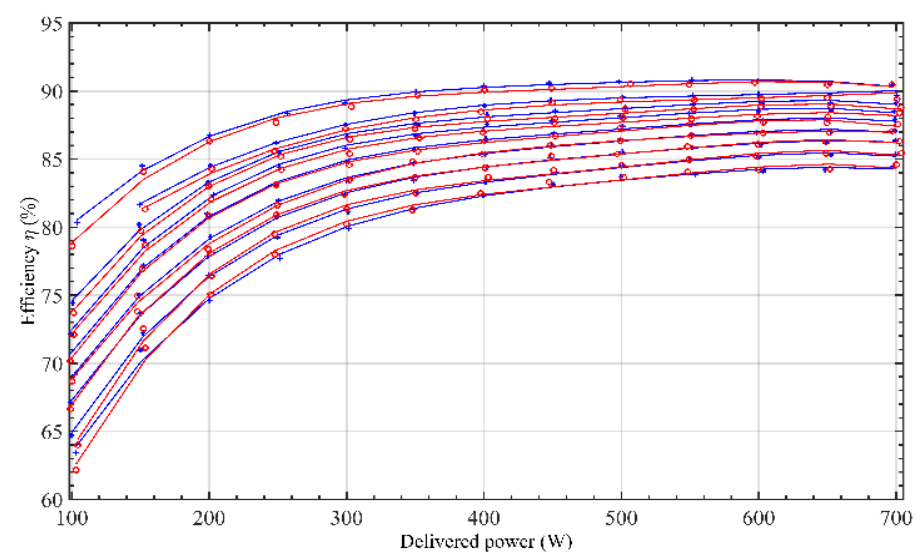

Fig. 11. Efficiency of the investigated system with inactive compensation (blue lines) and active compensation (red lines)

However, based on the presented results a serious drawback has been observed. In the presented system, the amount of losses generated in the H-bridge are decreased, however this increment is consumed by the additional semiconductor devices. In addition, the size of the system is increased which leads to power density drop.

\section{References}

[1] Boscaino V., Pellitteri F., Capponi G., La Rosa R., A wireless battery charger architecture for consumer electronics, Proc. of IEEE Second International Conference on Consumer Electronics, pp. 84-88 (2012).

[2] Achterberg J., Lomonova E.A., de Boeij J., Coil Array Structures Compared for Contactless Battery Charging Platform, IEEE Transactions on Magnetics, vol. 44, no. 5, pp. 617-622 (2008).

[3] Woronowicz K., Palka R., Optimised Thrust Control of Linear Induction Motors by a Compensation Approach, International Journal of Applied Electromagnetics and Mechanics, vol. 18, pp. 1-4 (2003).

[4] Woronowicz K., Palka R., An advanced linear induction motor control approach using the compensation of its parameters, Electromagnetic Fields in Electrical Engineering, vol. 22, p. 335 (2002).

[5] Stamati T.-E., Bauer P., On-road charging of electric vehicles, Proc. of IEEE Transportation Electrification Conference and Expo (ITEC), pp. 1-8 (2013).

[6] Chen L.J., Nagendra G.R., Boys J.T., Covic G.A., Double-coupled systems for roadway IPT systems, Proc. of IEEE Applied Power Electronics Conference and Exposition - APEC 2014, pp. 1618-1625 (2014).

[7] Onar O.C., Miller J.M., Campbell S.L., Coomer C., White C.P., Seiber L.E., A novel wireless power transfer for in-motion EV/PHEV charging, Proc. of 2013 Twenty-Eighth Annual IEEE Applied Power Electronics Conference and Exposition (APEC), pp. 3073-3080 (2013). 
[8] Prasanth V., Bauer P., Study of misalignment for On Road Charging, Proc. of IEEE Transportation Electrification Conference and Expo (ITEC), pp. 1-8 (2013).

[9] Chow J.P.W., Nan Chen, Chung H.S.H., Chan L.L.H., Misalignment tolerable coil structure for biomedical applications with wireless power transfer, Proc. of 35th Annual International Conference of the IEEE Engineering in Medicine and Biology Society (EMBC), vol. 2013, pp. 775-778 (2013).

[10] Villa J.L., Sallan J., Sanz Osorio J.F., Llombart A., High-Misalignment Tolerant Compensation Topology For ICPT Systems, IEEE Transactions on Industrial Electronics, vol. 59, no. 2, pp. 945-951 (2012).

[11] Zheng C., Chen R., Lai J., Design considerations to reduce gap variation and misalignment effects for inductive power transfer system, Proc. of IECON 2014, 40th Annual Conference of the IEEE Industrial Electronics Society, vol. 30, no. 11, pp. 1384-1390 (2014).

[12] Gati E., Kampitsis G., Stavropoulos I., Papathanassiou S., Manias S., Wireless Phase - Locked Loop control for inductive Power Transfer Systems, Proc. of IEEE Applied Power Electronics Conference and Exposition (APEC) pp. 1601-1607 (2015).

[13] Qiang H., Huang X., Tan L., Ji Q., Zhao J., Achieving maximum power transfer of inductively coupled wireless power transfer system based on dynamic tuning control, Science China Technological Sciences, vol. 55, no. 7, pp. 1886-1893 (2012).

[14] Zaheer M., Patel N., Hu A.P., Parallel tuned contactless power pickup using saturable core reactor, Proc. of IEEE International Conference on Sustainable Energy Technologies (ICSET), pp. 1-6 (2010).

[15] Si P., Hu A., Malpas S., Budgett D., Switching Frequency Analysis of Dynamically Detuned ICPT Power Pick-ups, Proc. of International Conference on Power System Technology, pp. 1-8 (2006).

[16] Hsu J.-U.W., Hu A.P., Swain A., A Wireless Power Pickup Based on Directional Tuning Control of Magnetic Amplifier, IEEE Transactions on Industrial Electronics, vol. 56, no. 7, pp. 2771-2781 (2009).

[17] Hu A.P., Hussmann S., Improved Power Flow Control for Contactless Moving Sensor Applications, IEEE Power Electronics Letters, vol. 2, no. 4, pp. 135-138 (2004).

[18] Marcinek M., Holub M., Coreless, Contactless Power Supply System with DSP Controlled Active Compensation of Parameter Changes, Elektronika ir elektrotechnika, vol. 20, no. 10, pp. 11-16 (2014).

[19] Kalisiak S., Marcinek M., Holub M., Palka R., Contactless power supply system with resonant circuit parameter change compensation, Proc. of 14th European Conference on Power Electronics and Applications (EPE 2011), pp. 1-8 (2011).

[20] Kalwar K.A., Aamir M., Mekhilef S., Inductively coupled power transfer (ICPT) for electric vehicle charging - A review, Renewable and Sustainable Energy Reviews, vol. 47, pp. 462-475 (2015).

[21] Lee J.-Y., Han B.-M., A Bidirectional Wireless Power Transfer EV Charger Using Self-Resonant PWM, IEEE Transactions on Power Electronics, vol. 30, no. 4, pp. 1784-1787 (2015).

[22] Safaee A., Woronowicz K., Time-Domain Analysis of Voltage-Driven Series-Series Compensated Inductive Power Transfer Topology, IEEE Transactions on Power Electronics, vol. 32, no. 7, pp. 4981-5003 2017.

[23] Erickson R.W., Maksimović D., Fundamentals of Power Electronics, Boston, MA: Springer US (2001).

[24] James J., Boys J., Covic G., A variable inductor based tuning method for ICPT pickups, Proc. of International Power Engineering Conference, vol. 2, pp. 1142-1146 (2005).

[25] Hsu J.U.W., Hu A.P., Determining the variable inductance range for an LCL wireless power pickup, IEEE Conference on Electron Devices and Solid-State Circuits 2007, EDSSC 2007, pp. 489-492 (2007).

[26] Mucko J., Strzelecki R., Errors in the analysis of series resonant inverter/converter assuming sinusoidal waveforms of voltage and current, Proc. of 2016 10th International Conference on Compatibility, Power Electronics and Power Engineering (CPE-POWERENG), pp. 369-374 (2016). 